\title{
Risk Factors for Clinical Coronary Heart Disease in Systemic Lupus Erythematosus: The Lupus and Atherosclerosis Evaluation of Risk (LASER) Study
}

\author{
SAHENA HAQUE, CAROLINE GORDON, DAVID ISENBERG, ANISUR RAHMAN, PETER LANYON, \\ AUBREY BELL, PAUL EMERY, NEIL McHUGH, LEE SUAN TEH, DAVID G.I. SCOTT, MOHAMED AKIL, \\ SOPHIA NAZ, JACQUELINE ANDREWS, BRIDGET GRIFFITHS, HELEN HARRIS, HAZEM YOUSSEF, \\ JOHN McLAREN, VERONICA TOESCU, VINODH DEVAKUMAR, JAMAL TEIR, and IAN N. BRUCE
}

\begin{abstract}
Objective. Accelerated atherosclerosis and premature coronary heart disease (CHD) are recognized complications of systemic lupus erythematosus (SLE), but the exact etiology remains unclear and is likely to be multifactorial. We hypothesized that SLE patients with CHD have increased exposure to traditional risk factors as well as differing disease phenotype and therapy-related factors compared to SLE patients free of CHD. Our aim was to examine risk factors for development of clinical CHD in SLE in the clinical setting.

Methods. In a UK-wide multicenter retrospective case-control study we recruited 53 SLE patients with verified clinical CHD (myocardial infarction or angina pectoris) and 96 SLE patients without clinical CHD. Controls were recruited from the same center as the case and matched by disease duration. Charts were reviewed up to time of event for cases, or the same "dummy-date" in controls. Results. SLE patients with clinical CHD were older at the time of event [mean (SD) 53 (10) vs 42 (10) yrs; p $<0.001]$, more likely to be male [11 (20\%) vs $3(7 \%)$; p $<0.001]$, and had more exposure to all classic CHD risk factors compared to SLE patients without clinical CHD. They were also more likely to have been treated with corticosteroids (OR 2.46; 95\% CI 1.03, 5.88) and azathioprine (OR 2.33; 95\% CI 1.16, 4.67) and to have evidence of damage on the pre-event SLICC damage index (SDI) (OR 2.20; $95 \%$ CI 1.09, 4.44). There was no difference between groups with regard to clinical organ involvement or autoantibody profile.

Conclusion. Our study highlights the need for clinical vigilance to identify modifiable risk factors in the clinical setting and in particular with male patients. The pattern of organ involvement did not differ in SLE patients with CHD events. However, the higher pre-event SDI, azathioprine exposure, and pattern of damage items (disease-related rather than therapy-related) in cases suggests that a persistent active lupus phenotype contributes to CHD risk. In this regard, corticosteroids and azathioprine may not control disease well enough to prevent CHD. Clinical trials are needed to determine whether classic risk factor modification will have a role in primary prevention of CHD in SLE patients and whether new therapies that control disease activity can better reduce CHD risk. (First Release Dec 1 2009; J Rheumatol 2010;37:322-9; doi:10.3899/jrheum.090306)
\end{abstract}

Key Indexing Terms:

SYSTEMIC LUPUS ERYTHEMATOSUS

CORONARY HEART DISEASE

RISK FACTORS

DISEASE ACTIVITY

From the arc Epidemiology Unit, School of Translational Medicine, University of Manchester; The Kellgren Centre for Rheumatology, Central Manchester and Manchester Children's University Hospitals NHS Trust, Manchester; Division of Immunity and Infection, University of Birmingham; Centre for Rheumatology, University College London; Department of Rheumatology, Queen's Medical Centre, Nottingham, UK; Department of Therapeutics and Pharmacology, Queen's University, Belfast, Ireland; Academic Unit of Musculoskeletal Disease, University of Leeds; Royal National Hospital for Rheumatic Diseases, Bath; Department of Rheumatology, Royal Blackburn Hospital; Department of Rheumatology, Norfolk and Norwich University Hospital; Sheffield Centre for Rheumatic Diseases, Royal Hallamshire Hospital, Sheffield; Department of Rheumatology, Northwick Park Hospital, The North West London Hospitals NHS Trust; Department of Rheumatology, Freeman Hospital, Newcastle; Department of Rheumatology, Cameron Hospital, Fife; Department of Rheumatology, Aberdeen Royal Infirmary; and Department of Rheumatology, Sunderland Royal Hospital.

Supported by a grant from Lupus UK. Additional support was obtained from the arc Epidemiology Unit core support grant. Prof. Bruce received support from The Manchester Academic Health Science Centre and The Manchester NIHR Biomedical Research Centre.

S. Haque, MRCP, arc Epidemiology Unit, University of Manchester; C. Gordon, MD, Division of Immunity and Infection, University of Birmingham; D. Isenberg, PhD, Centre for Rheumatology, University College Hospital London; A. Rahman, Medicine, University College London; P. Lanyon, PhD, Rheumatology, Queen's Medical Centre; A. Bell, MD, Rheumatology, Queen's University; P. Emery, MD, Academic Unit of Musculoskeletal Disease, Chapel Allerton Hospital; N. McHugh, MD, Rheumatology, Royal National Hospital for Rheumatic Diseases; L.S. Teh, MD, Rheumatology, Royal Blackburn Hospital; D.G.I. Scott, $M D$, Department of Rheumatology, Norfolk and Norwich University Hospital; M. Akil, MD; S. Naz, MD, Rheumatology, Royal Hallamshire Hospital; J. Andrews, MD, Rheumatology, Northwick Park Hospital; B. Griffiths, MD, Musculoskeletal Unit, Freeman Hospital; H. Harris, MD, Rheumatology, Cameron Hospital; H. Youssef, Rheumatology, Aberdeen Royal Infirmary; J. McLaren, MD, Rheumatology, Cameron 
Hospital; V. Toescu, MD, Rheumatology, Queen Elizabeth Hospital; V. Devakumar, MD, Rheumatology, Royal Blackburn Hospital; J. Teir, MD, Rheumatology, Sunderland Royal Hospital; I.N. Bruce, MD, FRCP, Professor and Honorary Consultant in Rheumatology, arc Epidemiology Unit, University of Manchester and Kellgren Centre for Rheumatology, Central Manchester and Manchester Children's University Hospitals NHS Trust.

Address correspondence to Dr. I.N. Bruce, arc Epidemiology Unit, School of Translational Medicine, The University of Manchester, Manchester UK M13 9PT.E-mail:ian.bruce@manchester.ac.uk

Accepted for publication August 31, 2009.

Accelerated atherosclerosis and premature coronary heart disease (CHD) are recognized complications of systemic lupus erythematosus (SLE) ${ }^{1}$. The pathogenesis of CHD in SLE appears to be a complex interaction of inflammatory, metabolic, and therapy-related factors, and those patients at high risk are difficult to identify. There is an increased prevalence of classic risk factors such as hypertension and diabetes mellitus among patients with $\mathrm{SLE}^{2,3}$. It is, however, argued that classic risk factors alone do not fully account for the burden of disease observed ${ }^{4}$. Additional factors associated with SLE such as inflammatory factors, prothrombotic states, renal disease, and the potential effects of lupus therapies are also believed to be relevant.

There are a large number of studies examining subclinical atherosclerosis in SLE using measures not widely available in the routine clinical setting. Only a few studies have specifically examined the risk factors for clinical events, of which a number include vascular events other than CHD, including stroke, peripheral vascular disease, or venous thromboembolism. It cannot, however, be assumed that these events will share the same precise pathological processes or precipitants ${ }^{1,5-9}$. As a result, findings of such studies have been variable and there is a lack of consistency across studies in factors identified.

We examined risk factors for the development of clinical CHD in the clinical setting in a multicenter UK network. The hypothesis tested was that patients with CHD have increased exposure to traditional risk factors, differing disease phenotype, and therapy-related factors compared to patients with SLE free of CHD.

\section{MATERIALS AND METHODS}

Patients. Thirteen UK centers from the British Isles Lupus Assessment Group (BILAG) and the British Society of Rheumatology Lupus Special Interest Group participated between August 2003 and July 2006. Rheumatologists identified SLE patients in their clinics using existing clinical or research databases. All subjects fulfilled the modified 1997 American College of Rheumatology (ACR) criteria for $\mathrm{SLE}^{10}$, which were verified by chart review at the time of data collection.

Cases. A case was defined as a patient with SLE who had a history of a first myocardial infarction (MI) or first diagnosis of angina pectoris after SLE diagnosis. MI was confirmed on the basis of 2 of the following 3: typical anterior/retrosternal chest pain; typical electrocardiographic changes or an elevation of cardiac enzymes (creatine kinase or troponin) ${ }^{11}$. Angina pectoris was defined as exertional or stress-related central chest pain relieved by rest or glyceryl trinitrate. In addition, confirmation of diagnosis of angi- na by a consultant cardiologist or by objective test such as a stress test or angiography was required.

Controls. For each case identified, 2 control subjects were recruited from the same center. The controls had no history of CHD and were matched for date of SLE onset (within 2 yrs) to enable matching for disease duration. Disease onset in all subjects was defined as the date that 4 ACR criteria were fulfilled ${ }^{10}$. Where more than 2 potential control subjects were identified for a case, random-number generation was employed to allow unbiased selection of 2 controls. We therefore matched cases and controls only on the basis of disease duration. This is because disease duration and duration of corticosteroid exposure are difficult to distinguish from each other as they are closely associated. By matching on disease duration we hoped to determine better the role of corticosteroid exposure to CHD risk.

Subjects were excluded if inadequate clinical information to confirm diagnosis of SLE and/or CHD was available from case note reviews or if they refused to provide informed consent. Sixty-one suitable cases were identified, of which 8 were excluded, and 121 controls were identified of which 25 were excluded (Figure 1). Two suitable controls were identified for 43/53 cases, and one control subject was identified for each of the 10 remaining cases because of (i) inability to match for disease duration, or (ii) because inadequate information was available from chart review, or (iii) the control subject declined to participate in the study. Therefore a total of 53 cases and 96 controls were recruited.

Study design. Data were collected by retrospective chart review using a predesigned form to standardize the information collected. Clinical and serological data were collected for each case up to the time of the coronary event. For matched controls we collected the same data up to a preassigned "dummy" date that was taken as the date of the event in their respective case (Figure 2).

Information collected included clinical features, laboratory observations (inflammatory markers, biochemistry, hematology, and autoantibody profile), the Systemic Lupus International Collaborating Clinics/American College of Rheumatology (SLICC/ACR) Damage Index (SDI) score ${ }^{12}$, and details of therapy exposure. The SDI was assessed in patients using information gathered from clinic note reviews up to the visit prior to diagnosis of the coronary event. Therefore the coronary event did not contribute to this score. Corticosteroid treatment was categorized as "previous use" or "never used," and the average daily dose was calculated where available. Details of immunosuppressive treatment were also collected. The presence of cardiovascular risk factors prior to the event or dummy date was noted.

Hypertension was defined as a systolic blood pressure of $>140 \mathrm{~mm} \mathrm{Hg}$ or diastolic $>90 \mathrm{~mm} \mathrm{Hg}$ or receiving treatment with an antihypertensive drug. Hypercholesterolemia was defined as total cholesterol $>5.2 \mathrm{mmol} / \mathrm{l}$, or receiving lipid-lowering therapy (when total cholesterol had not been recorded prior to an event, a value 3-6 months after the event was sought from the chart review and recorded where available). A positive family history of cardiovascular disease was defined as MI, angina, or sudden cardiac death in a first-degree relative: male $<55$ years or female $<60$ years of age Diabetes mellitus was defined as fasting plasma glucose $>7.0 \mathrm{mmol} / \mathrm{l}$ or current diabetic therapy. Smoking was recorded if the patient was noted in their medical record to have smoked prior to the clinical event or dummy date.

Clinical features of SLE such as malar rash, serositis, etc., were based on the physician's contemporaneous notes and clinic letters. In general each clinical feature or laboratory observation was noted as "ever present" and classified into organ systems as per the "classic" BILAG index ${ }^{13}$. Renal disease was defined as any patient with persistent proteinuria (> 500 $\mathrm{mg}$ /day), otherwise unexplained microscopic hematuria, chronic renal insufficiency, nephrotic syndrome, or any grade of verified lupus nephritis. For all cases followup data were also recorded, including recurrent coronary events, interventions undertaken, and vital status at the time of the study. Information was verified from additional primary or secondary care physicians as appropriate.

Statistical analysis and ethics. Data were analyzed using Stata 9.2 statisti- 


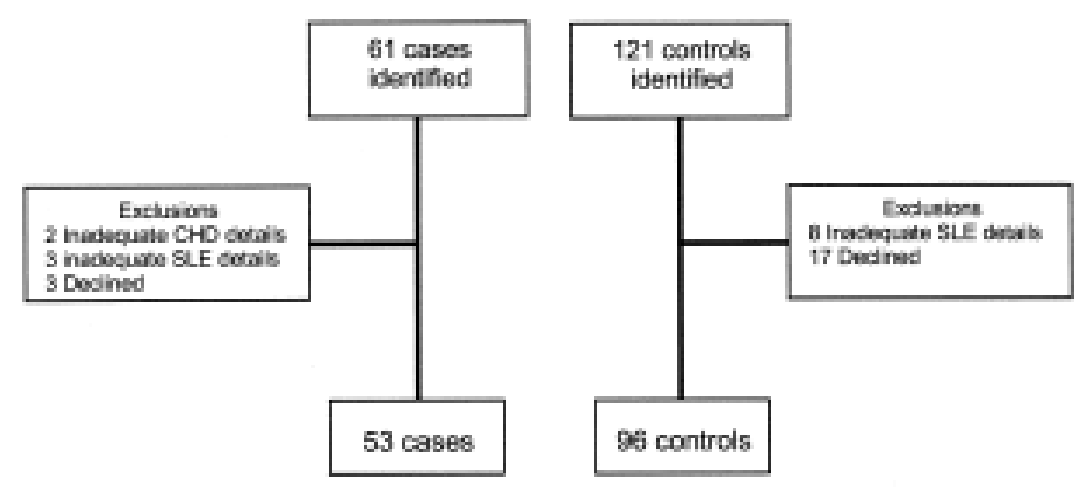

Figure 1. Selection of cases and controls. CHD: coronary heart disease; SLE: systemic lupus erythematosus.

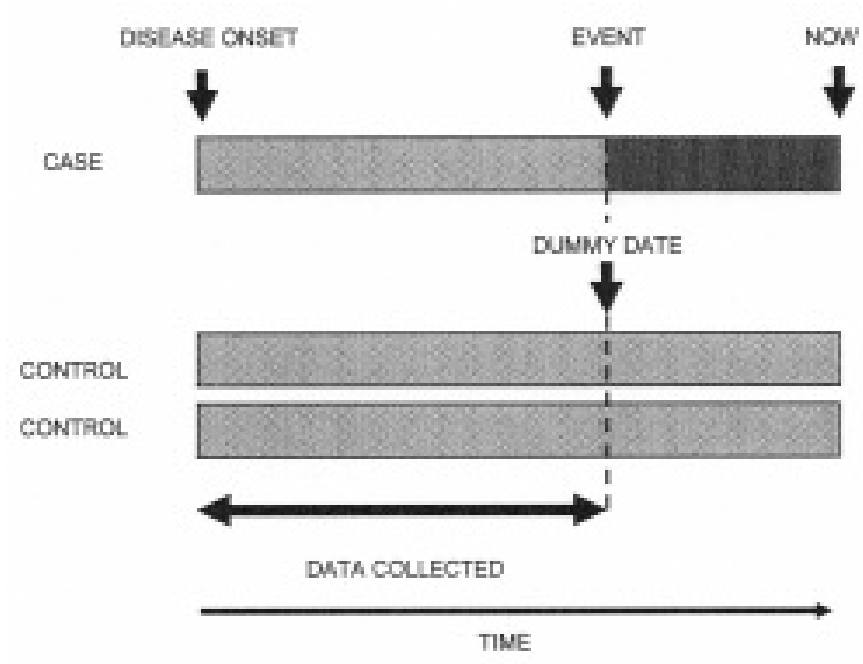

Figure 2. Overview of study design and data acquisition window for exposures in cases and controls.

cal software. Comparisons were made between cases and controls by means of a 2-sample t-test for continuous variables and by chi-square analysis for categorical variables. Two-sided $\mathrm{p}$ values $<0.05$ were considered to be significant. Logistic regression was used for multivariable analyses, with adjustment for disease duration to account for matching. There were some subjects in whom information was missing with regard to cardiovascular risk factors (details of hypertension missing for 1 case and 2 controls; hypercholesterolemia for 8 cases and 16 controls; family history of CHD for 14 cases and 22 controls; smoking status for 3 controls). For those variables where information was missing the analysis was undertaken using only those records that were complete, and raw data figures as well as the percentage are given where appropriate. The study was approved by the North-West Multicentre Research Ethics Committee (Reference number 03/8/012). Informed consent was obtained locally for all subjects. Data collection was permitted by the ethics committee for deceased patients from review of case records.

\section{RESULTS}

Cardiovascular events. Cases and controls were well matched for disease duration [mean (SD) 11 (8) vs 10 (8) yrs, respectively]. Of the 53 SLE patients with CHD, 23 $(43 \%)$ cases had an MI and $30(56 \%)$ had angina. Seven

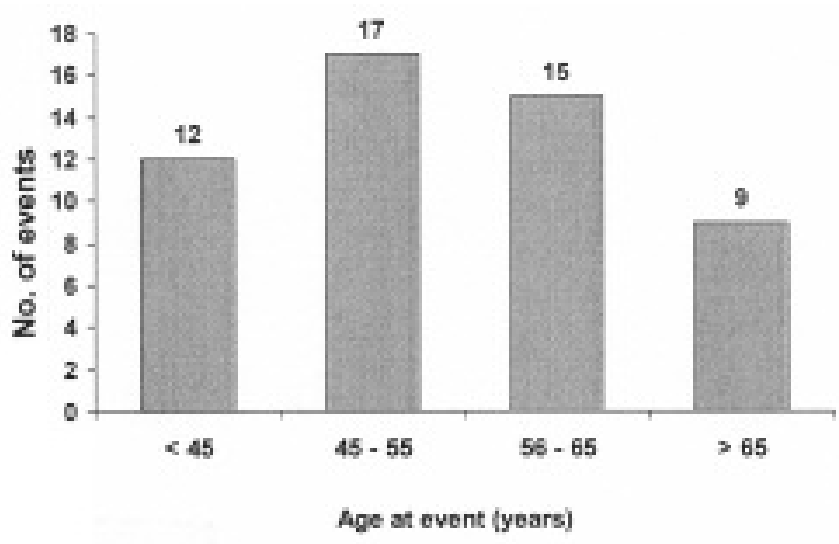

Figure 3. Distribution of age at onset of first coronary heart disease event in patients with systemic lupus erythematosus.

(32\%) cases with MI fulfilled 2 of 3 criteria and 15 (68\%) fulfilled all 3 criteria for MI. One additional patient presented with central crushing chest pain followed by sudden death attributed to MI. All cases with angina pectoris had a diagnosis verified by a consultant cardiologist, except one patient who presented to a general internist with documented chest pain characteristic of angina associated with a rise in creatine kinase and a minor rise in troponin, consistent with acute coronary syndrome but not MI. In view of the convincing clinical history of angina, supporting biochemical tests, and review by general internist, this case was included. Angina was confirmed using an objective confirmatory test in 24/30 patients. The mean (SD) age at the time of the first coronary event was 53 (10) years. The age at time of the event ranged from 33 to 73 years, and notably 12 (23\%) events occurred under age 45 years (Figure 3). Of 52 patients that survived the initial event, 12 (23\%) subsequently died over a mean (SD) of 8 (5) years. Eighteen (35\%) cases underwent coronary interventions; 12 had balloon angioplasty or coronary stent insertion and 6 underwent coronary revascularization. Seven patients $(39 \%$ of those with any intervention) underwent multiple interventions, although none had a second bypass graft. Of the 18 
patients that underwent an intervention, a successful outcome was achieved in $5(28 \%)$ patients, i.e., they remained symptom and medication-free at the time of study assessment. Of the remainder, 8 (44\%) patients continued on medication or remained symptomatic, 3 patients died, and the outcome is unknown in 2 patients.

Demographics. Cases were older than controls at the time of the event [mean (SD) 53 (10) vs 42 (10) yrs; p < 0.001) and were more likely to be male $[11(20 \%)$ vs $3(7 \%)$; p < $0.001)$. All further analyses were adjusted for age and gender. The other subject characteristics are described in Table 1. Prior to the CHD event, cases also had higher body weight compared to controls, and in the 25 cases and 38 controls in whom body mass index (BMI) could be calculated, the mean (SD) BMI was higher in cases [mean 28 (6) vs 25 (5); p $<0.01)$.

Classical CHD risk factors. All classic CHD risk factors examined occurred more frequently in cases compared to controls, and in the age and gender adjusted analysis, hypertension (adjusted OR 2.56, 95\% CI 1.05, 6.25) and a family history of premature CHD (adjusted OR 3.62, 95\% CI 1.15, 11.34) were associated with CHD events (Table 2). Only 2 subjects in the entire cohort had diabetes mellitus.

SLE organ involvement. In the whole population studied, the mean (SD) time from onset of autoimmune features (first SLE criterion) to fulfilling 4 ACR criteria was 4.8 (7.8) years. This time was significantly shorter for cases than con-

Table 1. Comparison of demographic and key lupus characteristics in cases and controls. All data are mean (SD) unless otherwise indicated.

\begin{tabular}{lccc}
\hline Characteristic & $\begin{array}{c}\text { Cases, } \\
\mathrm{n}=53\end{array}$ & $\begin{array}{c}\text { Controls, } \\
\mathrm{n}=96\end{array}$ & $\mathrm{p}$ \\
\hline Disease duration, yrs & $11(8)$ & $10(7)$ & - \\
Age at event time, yrs & $53(10)$ & $42(10)$ & $<0.001$ \\
Male, $\mathrm{n}(\%)$ & $11(20)$ & $3(7)$ & $<0.001$ \\
White, $\mathrm{n}(\%)$ & $46(88)$ & $71(77)$ & $\mathrm{NS}$ \\
Weight, $\mathrm{kg},{ }^{*} \mathrm{n}=94$ & $74(17)$ & $66(12)$ & 0.015 \\
\hline
\end{tabular}

* Total number of subjects for whom information was available for this variable if values were not available for entire group. NS: nonsignificant $\mathrm{p}$ value $>0.05$.

Table 2. Exposure of cardiovascular risk factors in SLE patients with coronary heart disease.

\begin{tabular}{lcc}
\hline Risk Factor, $\mathrm{n} *$ & $\begin{array}{c}\text { Unadjusted } \\
\text { OR }(95 \% \mathrm{CI})\end{array}$ & $\begin{array}{c}\text { Age and Gender-Adjusted } \\
\text { OR }(95 \% \mathrm{CI})\end{array}$ \\
\hline Hypertension, $\mathrm{n}=146$ & $3.52(1.65,7.54)$ & $2.56(1.05,6.25)$ \\
Hyperlipidemia, $\mathrm{n}=129$ & $3.91(1.57,9.71)$ & $3.06(0.99,9.52)$ \\
Smoker - ever, $\mathrm{n}=146$ & $1.89(1.06,2.72)$ & $1.54(0.52,2.56)$ \\
Family history, $\mathrm{n}=113$ & $3.04(1.23,7.53)$ & $3.62(1.15,11.34)$ \\
Body mass index, $\mathrm{n}=63$ & $1.14(1.03,1.27)$ & $1.05(0.91,1.21)$ \\
\hline
\end{tabular}

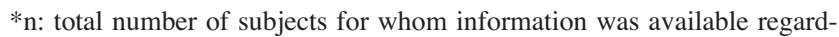
ing this variable if values were not available for the entire group. trols [mean (SD) 3.6 (6.4) vs 6.9 (9.5) yrs; $\mathrm{p}=0.02$ ). Cases and controls did not differ with regard to organ system involvement of their SLE. In particular, there were no statistically significant differences between the 2 groups with regard to inflammatory or valvular cardiac involvement. There were only 2 patients who ever had myocarditis and both were cases. The proportion of cases and controls with a history of verified lupus nephritis did not differ. In 33 cases and 63 controls for whom data were available, the maximum creatinine recorded at any time prior to the event date was significantly higher in cases [mean (SD) 137 (23) $\mu \mathrm{mol} / 1$ vs 92 (2) $\mu \mathrm{mol} / 1 ; \mathrm{p}=0.01]$. In the unadjusted analysis, cases were also more likely to have at least one item of damage scored on the SDI (OR 2.20; 95\% CI 1.09, 4.44; Table 3$)$. An exploratory analysis of the damage items noted that alopecia ( $1 \%$ vs $6 \%$; $\mathrm{p}=0.09)$, skin scarring ( $0 \%$ vs $4 \%$; $\mathrm{p}=0.05)$, premature gonadal failure (0\% vs $6 \% ; \mathrm{p}=0.02)$, and claudication ( 0 vs $8 \% ; \mathrm{p}=0.006)$ were all more frequent in cases than in controls.

Serology. The 2 groups were similar with regard to serology (Table 3). Notably, there was no significant difference in the proportion of cases and controls that had positive antiphospholipid antibodies (aPL) or lupus anticoagulant (LAC) (adjusted OR 2.6; 95\% CI 0.93, 7.1). There were no differences between cases and controls with regard to anti-dsDNA antibody (adjusted OR 0.76; 95\% CI 0.37 , 1.55 ) or anti-RNP antibody (unadjusted OR 1.04; $95 \% \mathrm{CI}$ $0.48,2.26)$ positivity.

Therapy. Cases were more likely to have had corticosteroid therapy (OR 2.46; 95\% CI 1.03, 5.88), although after adjustment this was no longer significant. Of the $111 / 149$ subjects

Table 3. Clinical, serological, and therapeutic exposures in SLE patients with coronary heart disease. Values in bold type are statistically significant.

\begin{tabular}{|c|c|c|}
\hline Risk Factor & $\begin{array}{l}\text { Unadjusted } \\
\text { OR }(95 \% \mathrm{CI})\end{array}$ & $\begin{array}{c}\text { Age and Gender } \\
\text { Adjusted } \\
\text { OR }(95 \% \mathrm{CI})\end{array}$ \\
\hline Renal disease & $0.79(0.37,1.67)$ & $1.26(0.50,3.20)$ \\
\hline Vasculitis* & $1.29(0.48,3.48)$ & $1.63(0.49,5.42)$ \\
\hline Neuropsychiatric disease & $0.86(0.43,1.71)$ & $0.95(0.41,2.21)$ \\
\hline SLICC Damage Index Score ${ }^{\dagger}$ & $2.20(1.09,4.44)$ & $1.73(0.73,4.11)$ \\
\hline $\begin{array}{l}\text { Antiphospholipid antibody or } \\
\text { lupus anticoagulant }\end{array}$ & $0.95(0.44,2.03)$ & $2.57(0.93,7.09)$ \\
\hline Anti-Sm antibody & $0.38(0.10,1.39)$ & $0.32(0.07,1.56)$ \\
\hline Anti-Ro antibody & $0.59(0.28,1.24)$ & $0.58(0.23,1.47)$ \\
\hline Anti-La antibody & $0.38(0.13,1.07)$ & $0.39(0.11,1.39)$ \\
\hline Corticosteroid & $2.46(1.03,5.88)$ & $2.63(0.97,7.16)$ \\
\hline Azathioprine & $2.33(1.16,4.67)$ & $3.18(1.33,7.59)$ \\
\hline Cyclophosphamide & $0.92(0.30,2.87)$ & $1.25(0.32,4.91)$ \\
\hline Methotrexate & $1.35(0.51,3.62)$ & $1.40(0.44,4.50)$ \\
\hline Cyclosporine & $0.75(0.19,3.05)$ & $1.22(0.22,6.69)$ \\
\hline Hydroxychloroquine & $1.13(0.54,2.39)$ & $1.11(0.46,2.66)$ \\
\hline
\end{tabular}

* Vasculitis defined as digital lesion, splinter hemorrhage, or peripheral gangrene. SLICC: Systemic Lupus International Collaborating Clinics. ${ }^{\dagger}$ At least one item scoring 
that were treated with steroids, doses were available for 89 subjects. The mean (SD) steroid dose for cases $(n=40)$ was 8.1 (10) $\mathrm{mg}$ and for controls $(\mathrm{n}=49) 9.1$ (12) $\mathrm{mg}$. The increased azathioprine exposure was, however, significant in both the unadjusted and adjusted analyses (adjusted OR $3.18 ; 95 \%$ CI 1.33, 7.59). In contrast, there were no differences in exposure to other immunosuppressive agents between the groups. Only 3 patients had been prescribed mycophenolate mofetil. With regard to other therapies, aspirin exposure was similar between the groups [20 (25\%) vs $13(21 \%) ; \mathrm{p}=0.37$ ], and a higher proportion of cases had been prescribed a lipid-lowering drug prior to the event date [7 (14\%) vs 2 (2\%); p $=0.005]$, consistent with the increased frequency of hypercholesterolemia.

\section{DISCUSSION}

In this UK-wide case-control study we found that SLE patients with clinical CHD were older, more likely to be male, and had more exposure to classic CHD risk factors. They were also more likely to have been treated with corticosteroids and azathioprine and have evidence of damage on the pre-event SLICC damage index. The older age at event is consistent with other studies that have shown an older age at time of diagnosis is associated with $\mathrm{CHD}^{1,14}$. The mean age at the time of first coronary event in this study was 53 years, which accords with the range of 47-51 years reported in previous studies ${ }^{1,6,7,9}$. Male patients were overrepresented in the cases, as noted by Petri, et $a l^{7}$. Overall, $20 \%$ of cases were male compared to $7 \%$ of controls. The control group is consistent with the expected background gender distribution of SLE in the $\mathrm{UK}^{15}$ and confirms the risk of CHD in men with SLE to be particularly increased.

Classic risk factors for $\mathrm{CHD}$, i.e., hypercholesterolemia, hypertension, smoking, and family history of CHD, were associated with clinical CHD; after adjustment for age and gender, hypertension and family history of CHD remained significantly associated with CHD events. These results are in keeping with 3 previous North American studies that used clinical CHD as an outcome ${ }^{1,6,7}$, and contrasts with studies that included other cardiovascular outcomes in addition to $\mathrm{CHD}^{5,8,16,17}$, where the contribution of classic risk factors is less clear (Table 4). This is because additional factors may contribute to the risk of other outcomes, e.g., atrial fibrillation and valvular heart disease are likely to also be important in stroke risk ${ }^{18-20}$; similarly, the hierarchy of risk factors for peripheral vascular disease also differs from $\mathrm{CHD}^{21}$. Our study confirms the findings of previous prospective cohort studies that classic risk factors play a key role in the devel-

Table 4. Summary of studies examining risk factors for clinical cardiovascular events in patients with SLE. Values are mean (range or \pm SD).

\begin{tabular}{|c|c|c|c|c|c|}
\hline Author & $\begin{array}{l}\text { Mean Age at Time } \\
\text { of Event, yrs }\end{array}$ & CHD Only & Cardiovascular Outcomes & Classic Risk Factors & Lupus/Other Factors \\
\hline Gladman $^{6}, \mathrm{n}=45$ & $48(25-73)$ & Yes & hyp & $\begin{array}{l}\text { Hypertension, congestive heart } \\
\text { failure, hypercholesterolemia, } \\
\text { pertriglyceridemia, hyperglycemia, } \\
\text { diabetes mellitus }\end{array}$ & Pericarditis, myocarditis \\
\hline $\operatorname{Manzi}^{1}, \mathrm{n}=33^{\dagger}$ & $48(22-72)$ & Yes & - & $\begin{array}{l}\text { Hypercholesterolemia, } \\
\text { postmenopausal status }\end{array}$ & $\begin{array}{l}\text { Older age at diagnosis*, longer } \\
\text { disease duration*, duration of } \\
\text { steroid use }\end{array}$ \\
\hline \multicolumn{2}{|c|}{ Svenungsson ${ }^{8}, \mathrm{n}=26$} & No & CHD, stroke, or PVD & $\begin{array}{c}\text { High VLDL, LDL, } \\
\text { lipoprotein a, low HDL }\end{array}$ & $\begin{array}{c}\text { ESR, CRP, orosomucoid, } \\
\alpha-1 \text {-antitrypsin, LAC, homocysteine, } \\
\text { osteoporosis, cumulative } \\
\text { steroid dose }\end{array}$ \\
\hline Petri $^{7}, \mathrm{n}=19$ & & Yes & - & $\begin{array}{l}\text { Hypercholesterolemia, } \\
\text { hypertension }\end{array}$ & $\begin{array}{l}\text { Older age at diagnosis, longer } \\
\text { disease duration, duration of } \\
\text { steroid use }\end{array}$ \\
\hline Bessant $^{16}, \mathrm{n}=29$ & & No & $\begin{array}{c}\text { "Survivors" only, stroke, } \\
\text { PVD }\end{array}$ & $\begin{array}{c}\text { Hypertension, high total } \\
\text { cholesterol, high triglycerides }\end{array}$ & LAC, less hydroxychloroquine use \\
\hline Freire $^{5}, n=10$ & 43 & No & CHD, stroke & Older age & $\begin{array}{l}\text { Longer disease duration, SLE } \\
\text { clinical features not assessed }\end{array}$ \\
\hline $\mathrm{Ho}^{17}, \mathrm{n}=42$ & & No & $\begin{array}{c}\text { CHD, stroke, PVD, venous } \\
\text { thrombosis }\end{array}$ & Smoking & $\begin{array}{l}\text { Mucocutaneous manifestations, } \\
\text { serosal manifestations, SDI, } \\
\text { SLAM, steroid therapy }\end{array}$ \\
\hline Urowitz $^{9}, \mathrm{n}=118$ & $51( \pm 12.3)$ & No & CHD, stroke, PVD & $\begin{array}{l}\text { Hypertension, smoking, } \\
\text { hypercholesterolemia, no. of } \\
\text { traditional risk factors }\end{array}$ & $\begin{array}{c}\text { Raynaud's, renal disease, } \\
\text { neuropsychiatric disease, vasculitis, } \\
\text { elevated prothrombin time, steroid } \\
\text { therapy/immunosuppressives, } \\
\text { less antimalarials }\end{array}$ \\
\hline
\end{tabular}

* Significant variables after controlling for age. ${ }^{\dagger}$ SLE cases vs non-SLE controls. CHD: coronary heart disease; PVD: peripheral vascular disease; LAC: lupus anticoagulant; ESR: erythrocyte sedimentation rate; CRP: C-reactive protein; VLDL: very low density lipoprotein; HDL: high density lipoprotein; SDI: SLICC Damage Index Score; SLAM: Systemic Lupus Activity Measure. 
opment of CHD in lupus patients ${ }^{7,22}$. A recent study has suggested that, although our recognition and treatment of classic risk factors such as hypertension and hypercholesterolemia has improved over time, a number of SLE patients eligible for treatment remain untreated ${ }^{23}$. One contributing factor for this observation may be the lack of any trials to delineate whether aggressive risk factor modification will reduce clinical events in SLE and the logistical difficulties of conducting such studies in the setting of $\mathrm{SLE}^{24}$. Our study, however, highlights the need for such intervention trials against clinical outcomes to answer this key question in SLE.

In agreement with others, we found that SLE-related factors are also important in CHD development ${ }^{8,9,17}$. The SLICC/ACR damage index (SDI) prior to the event showed a significant association with clinical CHD in the unadjusted analysis. It has been noted that patients who develop SLE at an older age accrue damage at a higher rate than SLE patients with a younger age of onset ${ }^{25}$ and hence the less significant result after age and gender adjustment. The main damage items that scored among cases were skin scarring, alopecia, premature gonadal failure, and claudication. Interestingly, few SDI items in these patients included factors directly attributable to the consequences of corticosteroid use, e.g., cataracts or osteoporosis, suggesting that SLE-associated disease activity and damage resulting from this may be important in the predisposition to CHD. Of note, the proportion of patients with lupus nephritis did not differ between cases and controls, and this finding is also consistent with previous studies ${ }^{1,6-8}$. Information regarding lupus nephritis was verified by chart review and biopsy reports in all cases. Importantly, however, cases had a higher peak serum creatinine "ever" compared to controls, suggesting that (as in the general population) renal impairment, regardless of etiology, is important to consider as a risk factor for $\mathrm{CHD}^{26}$.

Corticosteroid treatment and azathioprine were both associated with CHD, and azathioprine remained significant in our adjusted analysis. Azathioprine exposure has been shown to be associated with both subclinical and clinical vascular events in SLE ${ }^{17,27,28}$. This association may be explained in part by disease severity; however, other "severity" features such as vasculitis, nephritis, or neurological involvement were not significantly different between groups, and exposure to other immunosuppressive agents showed no significant differences between groups. The higher SDI and the association of clinical CHD with corticosteroids and azathioprine suggest that current therapies may not sufficiently control persistent grumbling disease activity to prevent irreversible damage in SLE. In addition, a recent study suggested that episodes of disease activity and episodes of higher steroid use both independently contribute to exacerbation of classic risk factors in SLE, which may provide a further mechanism by which persistent dis- ease and therapy together mediate cardiovascular risk in SLE $^{29}$.

A surprising finding is the lack of association between CHD events and aPL or LAC. Other studies using clinical outcomes have been inconsistent in this regard (Table 4). A lack of statistical power in most studies to date is the most likely explanation for this inconsistency. Others have found an association between LAC and cardiovascular disease outcomes in studies that include stroke, MI, and peripheral vascular disease ${ }^{8,16}$. There is also evidence that aPL may be pro- or anti-atherogenic ${ }^{27,30}$ and therefore measurement of specific aPL subtypes may have more predictive value than routine clinical tests.

Of the cases surviving their initial event 18 (35\%) subsequently underwent a coronary intervention including balloon angioplasty, coronary artery stent insertion, or revascularization. A favorable outcome was observed in one-third of these patients over a mean (SD) of 8 (5) years' followup. Although a few small case series have described an acceptable immediate outcome in patients with SLE undergoing coronary intervention, the long or medium term outcome in these patients remains unknown ${ }^{31-33}$.

It is important to consider the limitations of a study such as this. The association between SDI and azathioprine exposure is interesting; however, in view of the retrospective data collection, we lacked accurate disease activity measures over time on which to base any firm conclusions from these observations. The relatively small sample size is also a key limitation. Some difficulty was encountered collecting data because of missing information in medical records. For example, information was missing in up to $20 \%$ of subjects with regard to patient-assigned ethnicity, hyperlipidemia, and family history. However, we deliberately designed the study to match by center as we anticipated that the quality of data collection and missing information might be an issue, and our analysis suggested that data quality did indeed balance out between cases and controls according to the recruiting center. Complete data regarding most other variables were available in $98 \%$ or more of the subjects. To limit information bias, data were verified from other sources including cardiology and general practice records. In keeping with many studies, an accurate measure of corticosteroid exposure was difficult to ascertain owing to the retrospective nature of data collection. As a result, previous corticosteroid exposure of any length or dose appeared to be the most robust and verifiable measure and was therefore used in the analysis. As with any case-control study, bias introduced regarding case ascertainment and left censorship may be an issue. However, all participating centers had clinical or research databases allowing sampling from all eligible patients, including those that had died. Cases did have a shorter time from first criteria being met to diagnosis. This might have resulted in an underestimation of the exposure to immunosuppressant therapy in some subjects as data regard-

Personal non-commercial use only. The Journal of Rheumatology Copyright @ 2010 . All rights reserved. 
ing treatment were collected from the time of diagnosis, i.e., the time 4 ACR criteria were fulfilled. A longer delay to fulfilling criteria in the controls, however, would bias our results towards the null hypothesis. This observation of shorter time between onset of symptoms and diagnosis in cases with $\mathrm{CHD}$ is also consistent with the hypothesis that these patients had more aggressive active disease ${ }^{34}$.

To our knowledge, this is the largest study to date examining risk factors for verified $\mathrm{CHD}$ as a discrete clinical outcome in SLE. Our results confirm that classic risk factors and certain SLE-related characteristics are associated with an increased risk of $\mathrm{CHD}^{35}$. Our study highlights the importance of male gender and classic risk factors and the need for clinical vigilance to identify modifiable risk factors in the clinical setting. The higher pre-event SDI, azathioprine exposure prior to events, shorter time to diagnosis from symptom onset, and the pattern of damage items in cases suggest that a persistent active lupus phenotype contributes to $\mathrm{CHD}$ risk. In this regard, corticosteroids and azathioprine may not control disease well enough to prevent CHD. Evidence from clinical trials is now needed to resolve the question of whether classic risk factor modification will have a role in preventing clinical CHD events in SLE patients and to determine whether new therapies that better control disease activity can reduce the CHD risk.

\section{ACKNOWLEDGMENT}

The authors would like to acknowledge Donna Taylor-Fesler and Nicola Dale for their administrative support; and Dr. Kathleen McElhone, Alice Muir, Joanna Shelmerdine, and Margaret McQuilkin for help with data collection at individual study centers.

\section{REFERENCES}

1. Manzi S, Meilahn EN, Rairie JE, Conte CG, Medsger TA Jr, Jansen-McWilliams L, et al. Age-specific incidence rates of myocardial infarction and angina in women with systemic lupus erythematosus: comparison with the Framingham Study. Am J Epidemiol 1997;145:408-15.

2. Bruce IN, Urowitz MB, Gladman DD, Ibanez D, Steiner G. Risk factors for coronary heart disease in women with systemic lupus erythematosus: the Toronto Risk Factor Study. Arthritis Rheum 2003;48:3159-67.

3. Petri M, Spence D, Bone LR, Hochberg MC. Coronary artery disease risk factors in the Johns Hopkins Lupus Cohort: prevalence, recognition by patients, and preventive practices. Medicine Baltimore 1992;71:291-302.

4. Esdaile JM, Abrahamowicz M, Grodzicky T, Li Y, Panaritis C, du Berger R, et al. Traditional Framingham risk factors fail to fully account for accelerated atherosclerosis in systemic lupus erythematosus. Arthritis Rheum 2001;44:2331-7.

5. Freire BF, da Silva RC, Fabro AT, dos Santos DC. Is systemic lupus erithematosus a new risk factor for atherosclerosis? Arq Bras Cardiol 2006;87:300-6.

6. Gladman DD, Urowitz MB. Morbidity in systemic lupus erythematosus. J Rheumatol 1987;14 Suppl 13:223-6.

7. Petri M, Perez-Gutthann S, Spence D, Hochberg MC. Risk factors for coronary artery disease in patients with systemic lupus erythematosus. Am J Med 1992;93:513-9.

8. Svenungsson E, Jensen-Urstad K, Heimburger M, Silveira A, Hamsten A, de Faire U, et al. Risk factors for cardiovascular disease in systemic lupus erythematosus. Circulation 2001;104:1887-93.

9. Urowitz MB, Ibanez D, Gladman DD. Atherosclerotic vascular events in a single large lupus cohort: prevalence and risk factors. J Rheumatol 2007;34:70-5.

10. Hochberg MC. Updating the American College of Rheumatology revised criteria for the classification of systemic lupus erythematosus [letter]. Arthritis Rheum 1997;40:1725.

11. Gillum RF, Fortmann SP, Prineas RJ, Kottke TE. International diagnostic criteria for acute myocardial infarction and acute stroke. Am Heart J 1984;108:150-8.

12. Gladman D, Ginzler E, Goldsmith C, Fortin P, Liang M, Urowitz $\mathrm{M}$, et al. The development and initial validation of the Systemic Lupus International Collaborating Clinics/American College of Rheumatology damage index for systemic lupus erythematosus. Arthritis Rheum 1996;39:363-9.

13. Hay EM, Bacon PA, Gordon C, Isenberg DA, Maddison P, Snaith ML, et al. The BILAG index: a reliable and valid instrument for measuring clinical disease activity in systemic lupus erythematosus. Q J Med 1993;86:447-58.

14. Roman MJ, Shanker BA, Davis A, Lockshin MD, Sammaritano L, Simantov R, et al. Prevalence and correlates of accelerated atherosclerosis in systemic lupus erythematosus. N Engl J Med 2003;349:2399-406.

15. Johnson AE, Gordon C, Palmer RG, Bacon PA. The prevalence and incidence of systemic lupus erythematosus in Birmingham, England. Relationship to ethnicity and country of birth. Arthritis Rheum 1995;38:551-8.

16. Bessant R, Duncan R, Ambler G, Swanton J, Isenberg DA, Gordon $\mathrm{C}$, et al. Prevalence of conventional and lupus-specific risk factors for cardiovascular disease in patients with systemic lupus erythematosus: A case-control study. Arthritis Rheum 2006;55:892-9.

17. Ho KT, Ahn CW, Alarcon GS, Baethge BA, Tan FK, Roseman J, et al. Systemic lupus erythematosus in a multiethnic cohort (LUMINA): XXVIII. Factors predictive of thrombotic events. Rheumatology 2005;44:1303-7.

18. Goldstein LB, Adams R, Alberts MJ, Appel LJ, Brass LM, Bushnell $\mathrm{CD}$, et al. Primary Prevention of Ischemic Stroke: A Guideline From the American Heart Association/American Stroke Association Stroke Council: Cosponsored by the Atherosclerotic Peripheral Vascular Disease Interdisciplinary Working Group; Cardiovascular Nursing Council; Clinical Cardiology Council; Nutrition, Physical Activity, and Metabolism Council; and the Quality of Care and Outcomes Research Interdisciplinary Working Group: The American Academy of Neurology affirms the value of this guideline. Stroke 2006;37:1583-633.

19. Kitagawa Y, Gotoh F, Koto A, Okayasu H. Stroke in systemic lupus erythematosus. Stroke 1990;21:1533-9.

20. Futrell N, Millikan C. Frequency, etiology, and prevention of stroke in patients with systemic lupus erythematosus. Stroke 1989;20:583-91.

21. Chi YW, Jaff MR. Optimal risk factor modification and medical management of the patient with peripheral arterial disease. Catheter Cardiovasc Interv 2008;71:475-89.

22. Bruce IN, Urowitz MB, Gladman DD, Hallett DC. Natural history of hypercholesterolemia in systemic lupus erythematosus. J Rheumatol 1999;26:2137-43.

23. Urowitz MB, Gladman DD, Ibanez D, Berliner Y. Modification of hypertension and hypercholesterolaemia in patients with systemic lupus erythematosus: a quality improvement study. Ann Rheum Dis 2006;65:115-7.

24. Costenbader KH, Liang MH, Chibnik LB, Aizer J, Kwon H, Gall V, et al. A pravastatin dose-escalation study in systemic lupus erythematosus. Rheumatol Int 2007;27:1071-7. 
25. Maddison P, Farewell V, Isenberg D, Aranow C, Bae SC, Barr S, et al. The rate and pattern of organ damage in late onset systemic lupus erythematosus. J Rheumatol 2002;29:913-7.

26. Anavekar NS, McMurray JJ, Velazquez EJ, Solomon SD, Kober L, Rouleau JL, et al. Relation between renal dysfunction and cardiovascular outcomes after myocardial infarction. N Engl J Med 2004;351:1285-95.

27. Ahmad Y, Shelmerdine J, Bodill H, Lunt M, Pattrick MG, Teh LS, et al. Subclinical atherosclerosis in systemic lupus erythematosus (SLE): the relative contribution of classic risk factors and the lupus phenotype. Rheumatology 2007;46:983-8.

28. Doria A, Shoenfeld Y, Wu R, Gambari PF, Puato M, Ghirardello A, et al. Risk factors for subclinical atherosclerosis in a prospective cohort of patients with systemic lupus erythematosus. Ann Rheum Dis 2003;62:1071-7.

29. Karp I, Abrahamowicz M, Fortin PR, Pilote L, Neville C, Pineau $\mathrm{CA}$, et al. Recent corticosteroid use and recent disease activity: independent determinants of coronary heart disease risk factors in systemic lupus erythematosus? Arthritis Rheum 2008;59:169-75.
30. Nicolo D, Goldman BI, Monestier M. Reduction of atherosclerosis in low-density lipoprotein receptor-deficient mice by passive administration of antiphospholipid antibody. Arthritis Rheum 2003;48:2974-8.

31. Bossert T, Falk V, Gummert JF, Rahmel A, Mohr FW. Coronary artery bypass grafting in patients with systemic lupus erythematosus. Z Kardiol 2003;92:219-21.

32. Bozbuga N, Erentug V, Kaya E, Akinci E, Yakut C. Coronary artery bypass grafting in patients with systemic lupus erythematosus. J Card Surg 2004;19:471-2.

33. Ward MM. Outcomes of hospitalizations for myocardial infarctions and cerebrovascular accidents in patients with systemic lupus erythematosus. Arthritis Rheum 2004;50:3170-6.

34. Vila LM, Alarcon GS, McGwin G Jr, Friedman AW, Baethge BA, Bastian HM, et al. Early clinical manifestations, disease activity and damage of systemic lupus erythematosus among two distinct US Hispanic subpopulations. Rheumatology 2004;43:358-63.

35. Bruce IN. 'Not only...but also': factors that contribute to accelerated atherosclerosis and premature coronary heart disease in systemic lupus erythematosus. Rheumatology 2005;44:1492-502. 